\title{
Conditional Survival and Cure of Patients With Colon or Rectal Cancer: A Population-Based Study
}

\author{
Seyed M. Qaderi, MD'; Paul W. Dickman, PhD²; Johannes H.W. de Wilt, MD, PhD'; and
} Rob H.A. Verhoeven, $\mathrm{PhD}^{1,3}$

\begin{abstract}
Background: The increasing number of colorectal cancer (CRC) survivors need survival estimates that account for the time already survived. The aim of this population-based study was to determine conditional survival, cure proportions, and time-to-cure (TTC) of patients with colon or rectal cancer. Materials and Methods: All patients with pathologic stage I-III CRC treated with endoscopy or surgery, diagnosed and registered in the Netherlands Cancer Registry between 1995 and 2016, and aged 18 to 99 years were included. Conditional survival was calculated for those diagnosed before and after 2007. Cure proportions were calculated using flexible parametric models. Results: A total of 175,384 patients with pathologic stage I $(25 \%)$, II (38\%), or III disease $(37 \%)$ were included. Conditional 5 -year survival of patients with stage I, II, and III colon cancer having survived 5 years was $98 \%, 94 \%$, and $92 \%$, respectively. For patients with stage I-III rectal cancer, this was $96 \%, 89 \%$, and $85 \%$, respectively. Statistical cure in patients with colon cancer was reached directly after diagnosis (stage I) to 6 years (stage III) after diagnosis depending on age, sex, and disease stage. Patients with rectal cancer reached cure 0.5 years after diagnosis (stage I) to 9 years after diagnosis (stage III). In 1995, approximately $42 \%$ to $46 \%$ of patients with stage III colon or rectal cancer, respectively, were considered cured, whereas in 2016 this percentage increased to $73 \%$ to $78 \%$, respectively. Conclusions: The number of patients with $\mathrm{CRC}$ reaching cure has increased substantially over the years. This study's results provide valuable insights into trends of CRC patient survival and are important for patients, clinicians, and policymakers.
\end{abstract}

J Natl Compr Canc Netw 2020;18(9):1230-1237 doi: $10.6004 /$ jnccn. 2020.7568

${ }^{1}$ Department of Surgical Oncology, Radboud University Medical Center, Nijmegen, the Netherlands; ${ }^{2}$ Department of Medical Epidemiology and Biostatistics, Karolinska Institute, Stockholm, Sweden; and ${ }^{3}$ Department of Research \& Development, Netherlands Comprehensive Cancer Organization, Utrecht, the Netherlands.

\section{Background}

Colorectal cancer (CRC) is the third most common cancer in the world. ${ }^{1,2}$ Survival of patients with CRC has improved significantly because of earlier diagnosis and better treatment of the primary tumor and metastatic disease. ${ }^{3}$ Survival estimates of patients with cancer based on data collected by population-based cancer registries are commonly determined using relative survival methods, with survival reported as a function of time since diagnosis. Excess mortality resulting from CRC is highest in the first year after diagnosis, especially in the geriatric population. ${ }^{4}$ Thus, published estimates of survival provide an overly pessimistic prognosis for patients who have survived an initial period after diagnosis. Alternative approaches to reporting patient survival, such as conditional survival and cure models, have recently been applied in the field of CRC. ${ }^{5,6}$ Conditional survival is the probability of surviving an additional time, given the patient has already survived some fixed time after diagnosis. ${ }^{7}$ Conditional survival is of interest for planning and designing more patient-centered follow-up schedules by healthcare professionals. In addition, it provides patient prognostic information based on the amount of time they have already survived since diagnosis and may increase their insurability. ${ }^{8}$ For many cancers, including $\mathrm{CRC}$, the all-cause mortality of patients will return to the level of the general population at some time during follow-up (ie, excess mortality will tend to zero). When this point is reached, the patients (as a group) alive are considered statistically cured. The time between diagnosis and this cure point is called time-to-cure (TTC). Cure modeling has been described in statistical literature, but has only recently gained popularity in cancer research. ${ }^{6,7}$ In cancers with long-term survivors, cure models can be a good way to characterize and study patients' survival. ${ }^{6,9,10}$ Some studies have reported longterm conditional survival outcomes, but mostly based on older data. ${ }^{5,11,12}$ There is an growing need for up-to-date and specific survival outcome information for the increasing number of CRC survivors. The aim of this population-based study was to report relative survival and 5-year conditional relative survival of patients diagnosed between 
1995 and 2016. In addition, cure proportions and TTC were modeled.

\section{Materials and Methods}

\section{Study Design and Data Collection}

This retrospective cohort study was conducted using data from the Netherlands Cancer Registry (NCR). ${ }^{13}$ All patients newly diagnosed with cancer are registered in the nationwide NCR. Information regarding patient and tumor characteristics are extracted directly from medical records by trained NCR data managers. Information on vital status at the end of February 2019 was obtained through annual linkage with the Municipal Personal Records Database. There were no patients included based on death certificate-only information.

\section{Patient Selection}

All patients with pathologic stage I-III colon or rectal cancer diagnosed and endoscopically or surgically treated between January 1995 and December 2016 in the Netherlands were included $(\mathrm{N}=178,384)$. Patients treated without surgery were excluded because resection continues to be the gold standard for curative treatment, and nearly all patients undergo resection. The mean age of patients treated without surgery was significantly higher than those undergoing resection (75.2 vs 69.3 years; chisquare test, $P<.001$ ).

If patients had multiple diagnosed CRCs, only the first diagnosed cancer was selected. In case of multiple simultaneous CRCs, the cancer with the highest pathologic stage or prognostically worst histology type was selected for analysis. Tumor localization was categorized using the ICD-O into colon (C18.0-18.9) and rectum (C19.9-20.9). Disease stage was based on the pathologic TNM classification according to the edition used at time of diagnosis (edition 4.2 for 1993-1998, the 5 th edition for 1999-2002, edition 6 for 2003-2009, and the 7 th edition for 2010-2019). Because there were only limited changes in TNM classification over time, no corrections were made. Information about age, sex, disease stage, and treatments were complete for all included patients.

\section{Statistical Analysis}

Net survival is a measure of patient survival in which deaths resulting from other causes are removed. Net survival can be estimated in either a relative survival or a cause-specific (also called disease-specific) framework. ${ }^{7}$ A cause-specific framework requires accurate information on cause of death, which is not always available with cancer registry data and in the case of the NCR. The relative survival framework facilitates estimation of

\begin{tabular}{|c|c|c|}
\hline & $\begin{array}{c}\text { Colon Cancer } \\
\text { n (\%) }\end{array}$ & $\begin{array}{c}\text { Rectal Cancer } \\
\text { n (\%) }\end{array}$ \\
\hline Total, $\mathrm{n}$ & 117,530 & 57,854 \\
\hline \multicolumn{3}{|l|}{ Sex } \\
\hline Male & $59,512(50.6)$ & $34,744(60.1)$ \\
\hline Female & $58,018(49.4)$ & 23,110 (39.9) \\
\hline \multicolumn{3}{|l|}{ Age, mean (SD), y } \\
\hline Male & $69.5(10.7)$ & $66.9(10.6)$ \\
\hline Female & $71.1(11.4)$ & $67.7(11.9)$ \\
\hline \multicolumn{3}{|l|}{ Year of diagnosis } \\
\hline 1995-1999 & $20,101(17.1)$ & $10,636(18.4)$ \\
\hline 2000-2004 & $22,505(19.1)$ & $12,183(21.1)$ \\
\hline 2005-2009 & $26,892(22.9)$ & $13,786(23.8)$ \\
\hline $2010-2014$ & $31,974(27.2)$ & $14,653(25.3)$ \\
\hline 2015-2016 & $16,058(13.7)$ & $6,596(11.4)$ \\
\hline \multicolumn{3}{|l|}{ Pathologic stage } \\
\hline 1 & $26,788(22.8)$ & $17,204(29.7)$ \\
\hline ॥ & $50,233(42.7)$ & $17,027(29.4)$ \\
\hline III & $40,509(34.5)$ & $23,623(40.9)$ \\
\hline \multicolumn{3}{|l|}{ Histology of primary tumor } \\
\hline Nonmucinous adenocarcinoma & $98,977(84.2)$ & $52,853(91.4)$ \\
\hline Mucinous adenocarcinoma & $16,892(14.4)$ & $4,616(8.0)$ \\
\hline Signet cell carcinoma & $1,154(1.0)$ & $261(0.5)$ \\
\hline NOS & $507(0.4)$ & $124(0.2)$ \\
\hline \multicolumn{3}{|l|}{ Systemic chemotherapy alone } \\
\hline No & $90,802(77.8)$ & $22,312(89.7)$ \\
\hline Yes & $25,951(22.2)$ & $2,567(10.3)$ \\
\hline \multicolumn{3}{|l|}{ Radiotherapy alone } \\
\hline No & $90,802(99.6)$ & $22,312(51.4)$ \\
\hline Yes & $409(0.4)$ & $21,077(48.6)$ \\
\hline \multicolumn{3}{|l|}{ Chemoradiation therapy } \\
\hline No & $117,162(99.7)$ & $45,956(79.4)$ \\
\hline Yes & $368(0.3)$ & $11,898(20.6)$ \\
\hline
\end{tabular}

Abbreviation: NOS, not otherwise specified.

the same quantity by incorporating expected probabilities of dying due to causes other than cancer estimated from general population life tables. We calculated relative survival using the Ederer II method up to 24 years after diagnosis using Dutch sex-, age-, and year-specific population life tables, and grouped patients into age categories of $<60$ and $\geq 60$ years. ${ }^{14,15}$ Incidence periods between 1995 and 2007 and 2008 and 2016 were defined. For each value of follow-up time from 0 (diagnosis) to 10 years, in half-year intervals, we used the same approach to estimate conditional 5-year relative survival. That is, we first estimated 5-year relative survival from diagnosis 
A

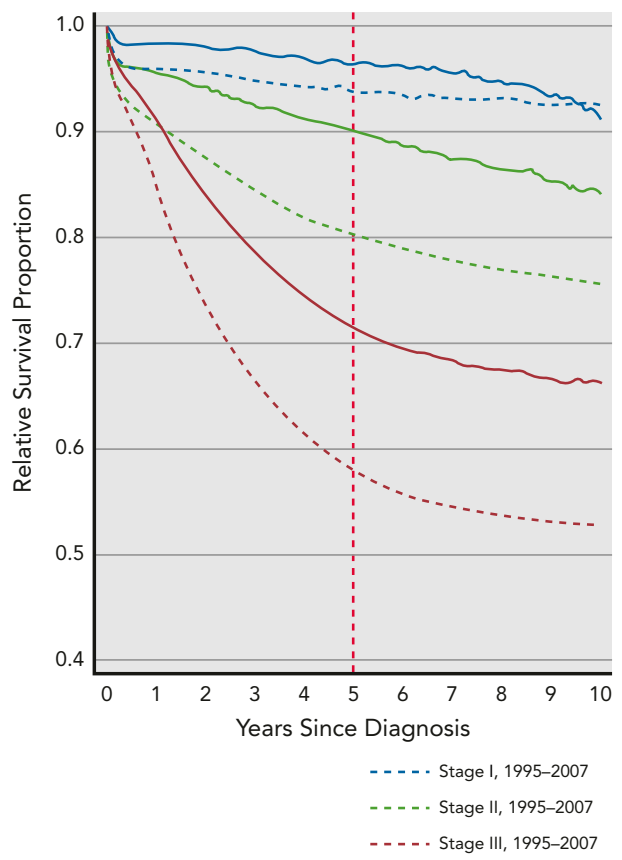

B

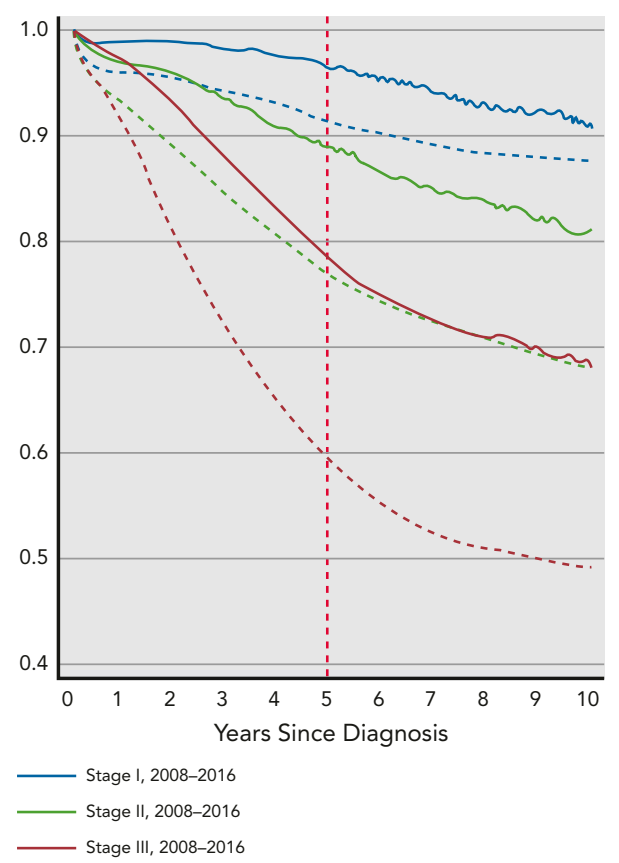

Figure 1. Relative survival (according to the Ederer II method) of patients with (A) colon or (B) rectal cancer, per stage, for both 1995-2007 and 2008-2016 cohorts.

(traditional 5-year relative survival). We then restricted the time at risk so that all patients first became at risk 0.5 years after diagnosis, and estimated relative survival for the interval $(0.5,5.5)$. That is, we imposed late entry. Patients who died or were censored within the first 0.5 years were excluded from the analysis. We then restricted the follow-up again so that all patients first became at risk 1 year after diagnosis, and estimated relative survival for the interval $(1,6)$, and so forth. The approach used for estimating conditional survival is illustrated elswere. ${ }^{16}$ When conditional survival exceeded $95 \%$, patients were considered as having minimal excess mortality compared with the general population, which is a commonly used definition for statistical cure. ${ }^{17,18}$ For the life table estimates, follow-up was divided into intervals of 0.1 years. The conditional relative survival estimates were calculated with half-year splits.

TTC was defined as the time until conditional 5 -year survival reached $95 \%$. In addition, cure proportions and median survival of the uncured were calculated using flexible parametric survival models. We chose to analyze the data in the framework of flexible parametric models, because these models are flexible enough to capture high excess hazard within the first period after diagnosis, which is common among the aged population. In the past, older patients were excluded to calculate accurate estimates with conventional parametric models. ${ }^{6}$ The cure proportion was defined as the proportion of patients estimated to experience mortality equal to the general population. Stata version 14 (StataCorp LLP) was used for analysis. ${ }^{19-21}$ We adhered to the STROBE checklist for observational cohort studies. ${ }^{22}$

\section{Results}

A total of 175,384 patients with pathologic stage I (25\%), II (38\%), and III (37\%) CRC were included. More than half of the patients were male (54\%) and two-thirds (67\%) had colon cancer. Systemic chemotherapy was administered in $22 \%$ of the patients with colon cancer and $10 \%$ of the those with rectal cancer, often as part of a combined chemoradiation regimen. Radiation therapy alone was given to $0.4 \%$ of the patients with colon cancer and $49 \%$ of those with rectal cancer. An overview of patient and tumor characteristics is provided in Table 1.

The 5-year relative survival of patients diagnosed with stage I, II, and III colon cancer between 2008 and 2016 was $96 \%, 90 \%$, and $71 \%$, respectively (Figure 1). For patients with rectal cancer, 5-year relative survival was $96 \%$ (stage I), $89 \%$ (stage II), and $78 \%$ (stage III). Relative survival of patients recently diagnosed with CRC has increased substantially compared with those diagnosed and treated between 1995 and 2007. This increase was most evident for patients with stage II or III disease. 
Table 2. Five-Year Relative Survival Conditional $(N=175,384)$

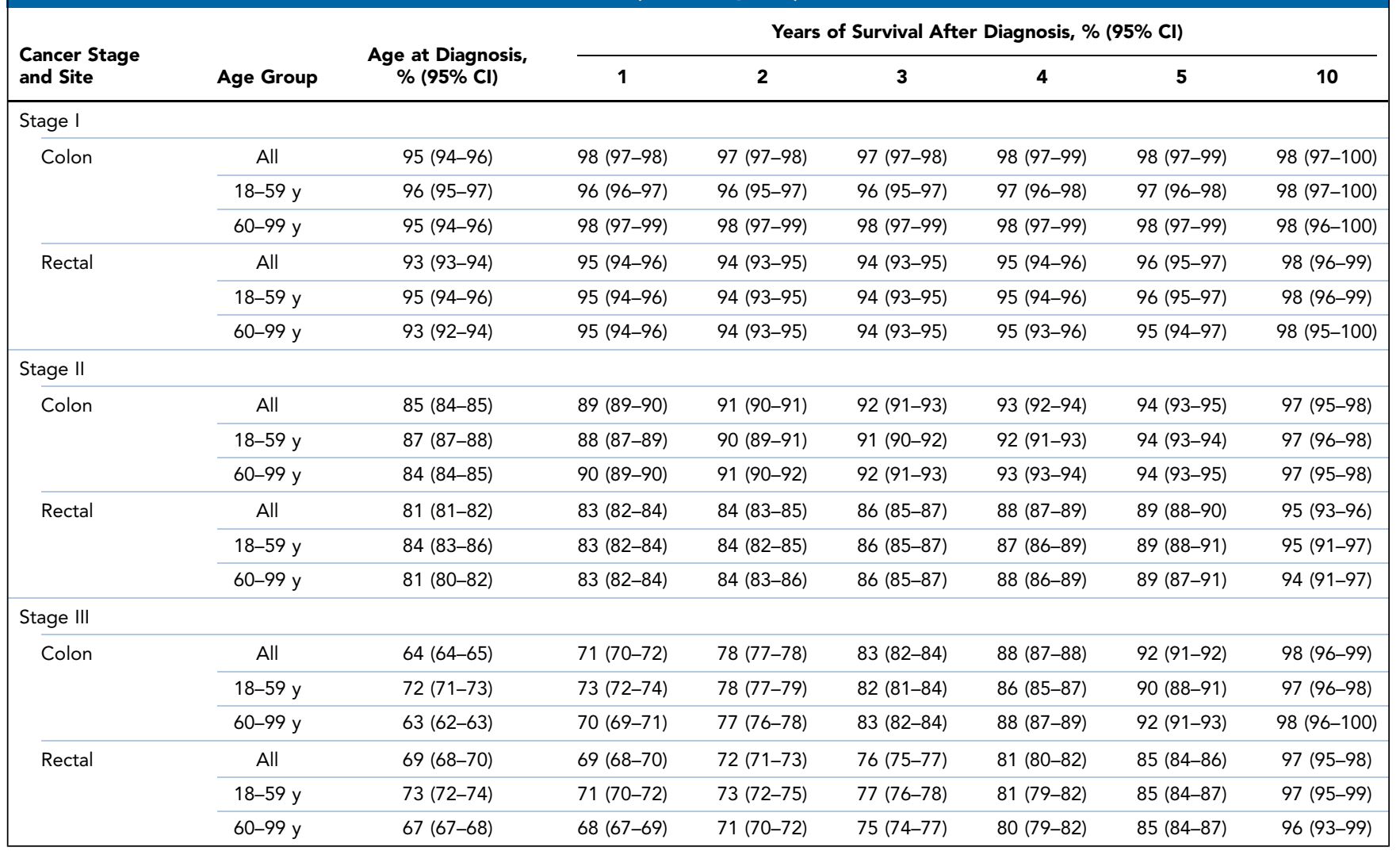

Follow-up available up to 2019.

\section{Conditional Survival and TTC}

Conditional survival increased with each additional year survived up to 10 years postoperatively. The 5-year relative survival for tumor site, stage, and age group conditional on 1,5 , and 10 years from diagnosis is presented in Table 2. Survival outcomes differed between stages, with stage III having lowest conditional survival. Conditional survival has increased in the past 2 decades, being higher in patients diagnosed between 2008 and 2016 (Figures 2 and 3). Better conditional survival was also seen for patients with colon cancer compared with those with rectal cancer for all stages and age groups. These differences diminished 10 years after diagnosis. Results are presented per stage for patients with colon or rectal cancer diagnosed between 1995 and 2016.

\section{Stage I}

Five-year relative survival for colon cancer conditional on having survived 1,5 , and 10 years was consistently $98 \%$. After 1-, 5-, and 10-year follow-up, patients with rectal cancer had 5 -year relative percentages of $95 \%, 96 \%$, and $98 \%$.

Both male and female patients with colon cancer, irrespective of age, reached (statistical) cure directly after diagnosis (TTC, 0 years) (Table 3). Patients with rectal cancer aged 60 to 99 years reached cure at 0.5 years, whereas those aged 18 to 59 years were cured directly after diagnosis.

\section{Stage II}

Conditional survival of colon cancer increased from $89 \%$ at 1 year, to $94 \%$ and $97 \%$ after having survived 5 and 10 years, respectively. Patients with rectal cancer alive at 1 , 5 , and 10 years after diagnosis had $83 \%, 89 \%$, and $95 \%$ chance of being alive another 5 years.

Male patients with colon cancer reached the cure point at approximately 6.5 years after diagnosis, whereas TTC for female patients was 4.5 years.

TTC for female patients with rectal cancer was 9.5 years. Conditional survival of male patients with stage II rectal cancer did not exceed $95 \%$ within our follow-up time.

\section{Stage III}

Conditional 5-year relative survival of colon cancer increased from $71 \%$ at 1 year, to $92 \%$ and $98 \%$ after having survived 5 and 10 years, respectively. Patients with stage III rectal cancer alive at 1,5 , and 10 years after diagnosis had $69 \%, 85 \%$, and $97 \%$ chance of being alive another 5 years. 
A

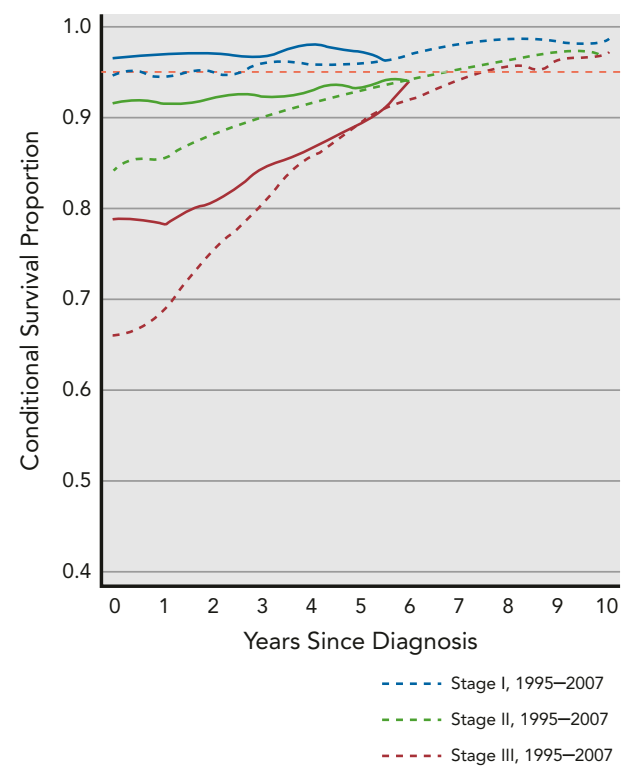

B

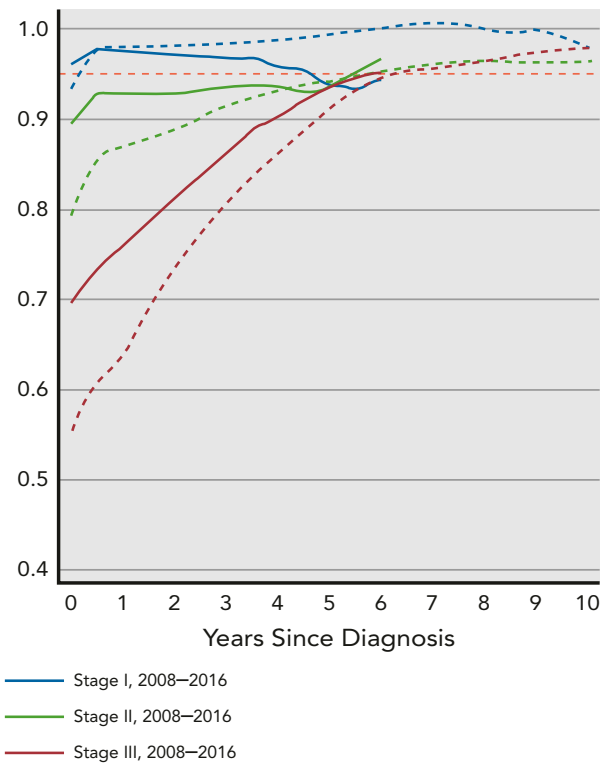

Figure 2. Conditional 5-year relative survival for every year survived after diagnosis of colon cancer stage stratified by incidence year interval (1995-2007 and 2008-2016) and age group: (A) 18-59 years and (B) 60-99 years. Conditional relative survival estimates were only possible up to 6 years for the 2008-2016 cohort (follow-up until 2019).

Cure was reached 7 and 6 years after diagnosis for male and female patients with colon cancer, respectively. Patients with rectal cancer were considered cured 9 (men) and 8.5 (women) years after diagnosis.

\section{A}

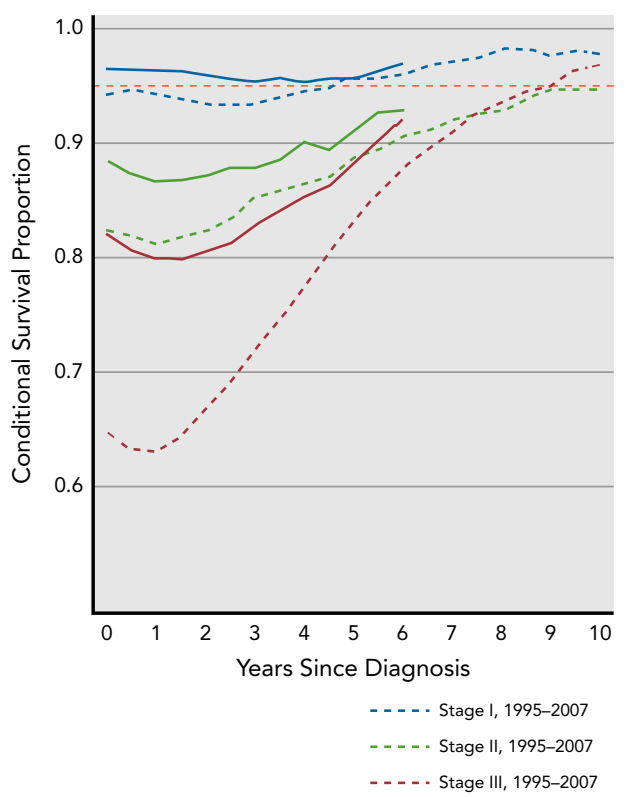

Cure Proportion and Survival of the Uncured The proportion of patients without excess mortality compared with the general population has increased. Cure proportions for patients aged 18 to 59 years with stage I, II,
B

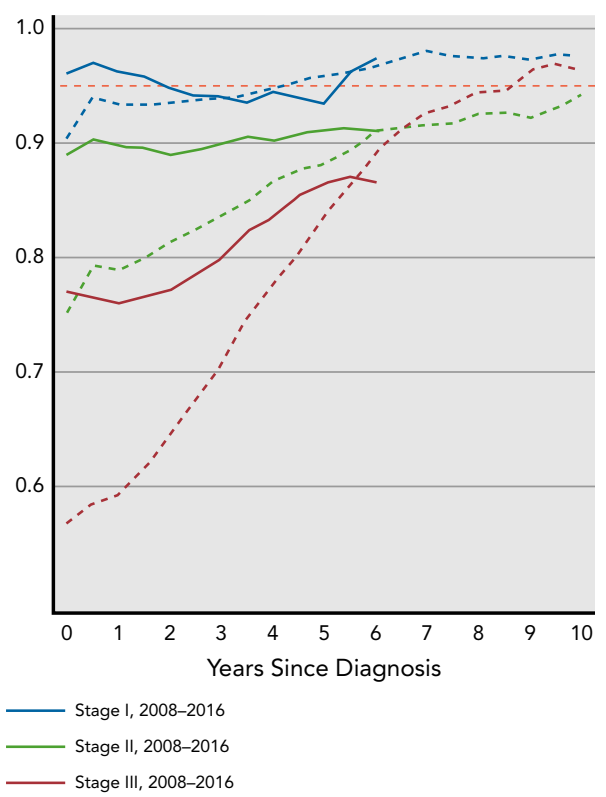

Figure 3. Conditional 5-year relative survival for every year survived after diagnosis of rectal cancer stage stratified by incidence year interval (1995-2007 and 2008-2016) and age group: (A) 18-59 years and (B) 60-99 years. Conditional relative survival estimates were only possible up to 6 years for the 2008 to 2016 cohort (follow-up until 2019). 


\begin{tabular}{|c|c|c|c|c|}
\hline Cancer Stage and Site & Age Group & Overall & Men & Women \\
\hline \multicolumn{5}{|l|}{ Stage I } \\
\hline \multirow[t]{3}{*}{ Colon } & All & 0 & 0 & 0 \\
\hline & $18-59$ y & 0 & 0 & 0 \\
\hline & $60-99$ y & 0 & 0 & 0 \\
\hline \multirow[t]{3}{*}{ Rectal } & All & 0.5 & 0.5 & 0.5 \\
\hline & $18-59$ y & 0 & 0 & 0 \\
\hline & $60-99$ y & 0.5 & 0.5 & 0.5 \\
\hline \multicolumn{5}{|l|}{ Stage II } \\
\hline \multirow[t]{3}{*}{ Colon } & All & 5.5 & 6.5 & 4.5 \\
\hline & $18-59$ y & 6 & 7 & 6 \\
\hline & $60-99$ y & 5.5 & 6 & 4 \\
\hline \multirow[t]{3}{*}{ Rectal } & All & 10 & $\mathrm{~N} / \mathrm{A}^{\mathrm{a}}$ & 9.5 \\
\hline & $18-59$ y & 9 & $\mathrm{~N} / \mathrm{A}^{\mathrm{a}}$ & 7.5 \\
\hline & $60-99$ y & $\mathrm{N} / \mathrm{A}^{\mathrm{a}}$ & $\mathrm{N} / \mathrm{A}^{\mathrm{a}}$ & 10 \\
\hline \multicolumn{5}{|l|}{ Stage III } \\
\hline \multirow[t]{3}{*}{ Colon } & All & 6.5 & 7 & 6 \\
\hline & $18-59$ y & 7.5 & 7.5 & 7 \\
\hline & $60-99$ y & 6 & 7 & 5.5 \\
\hline \multirow[t]{3}{*}{ Rectal } & All & 9 & 9 & 8.5 \\
\hline & $18-59$ y & 8.5 & 9 & 8.5 \\
\hline & $60-99$ y & 9 & 9 & 8 \\
\hline
\end{tabular}

Abbreviation: N/A, not applicable.

${ }^{a}$ Conditional survival $>95 \%$ not reached within available follow-up time.

and III colon cancer were $0.88,0.70$, and 0.46 , respectively, in 1995. In 2016, cure proportions had increased to 0.96, 0.88 , and 0.78 for stage I, II, and III, respectively. Cure proportions for older patients were lower (Figure 4).

Moreover, cure proportions of patients with rectal cancer aged 18 to 59 years have also increased. In 1995, cure proportions were 0.86 (stage I), 0.67 (stage II), and 0.42 (stage III). In 2016, cure proportions were $0.95,0.87$, and 0.73 , respectively. Patients aged 60 to 99 years again had lower cure proportions.

Median survival of patients with stage I colon cancer aged 18 to 59 years not reaching cure (ie, having excess mortality compared with the general population) has increased from 3.3 years in 1995 to 4.4 years in 2016 (Figure 4). For stage II and III, median survival of the uncured has increased from 3.0 and 2.7 years in 1995 to 4.3 and 4.2 years in 2016. Uncured patients with stage I-III rectal cancer had increased median survival over the past 2 decades, both for patients aged 18 to 59 and 60 to 99 years.

\section{Discussion}

This study presented up-to-date relative survival, conditional survival, cure proportions, and TTC estimates of patients with stage I-III CRC. Relative survival of patients with colon or rectal cancer has increased substantially over the years, and the increment is highest in more-advanced disease stages. These improvements in survival are in line with previous findings in the literature.,23 The 5-year relative survival conditional on the number of years survived has increased as well. Conditional survival outcomes of patients recently diagnosed with CRC were better compared with those diagnosed between 1995 and 2007. For patients with stage I, II, and III colon or rectal cancer, conditional survival improved with years survived and was higher in colon cancer compared with rectal cancer. This holds, in particular, for more advanced disease (stage II-III).

The largest improvements in conditional survival for patients with colon or rectal cancer were seen in older patients and during the first years after diagnosis. This is in line with a recent observation by our group in which we observed that especially elderly patients have an improved postoperative survival in the first years. ${ }^{4}$ The gap in survival difference has narrowed in recent years, and elderly patients (aged 60-99 years) now have an almost similar survival after treatment with curative intent compared with younger patients (aged 18-59 years). Conditional survival of women was slightly higher than that of men, and statistical cure was reached earlier than in male patients. TTC was longer for advanced disease stages. Among patients with stage I rectal cancer, younger patients were considered cured earlier. In contrast, older patients with stage II-III colon cancer had higher conditional survival and shorter TTC estimates than younger patients with a similar stage. All of these findings are in line with comparable studies. ${ }^{12}$ Patients with stage II rectal cancer did not reach conditional survival $>95 \%$ until 9 to 10 years after diagnosis. Despite being peculiar, this finding has been noted previously. ${ }^{5,11}$ Perhaps this patient group experienced long-term excess mortality because of disease recurrence. It is likely that excess mortality among older patients decreases faster over time compared with younger patients. ${ }^{11}$ Within the relative survival framework, the impact of mortality is less dominating because of limited life expectancy in the elderly. In general, cure proportions have increased substantially over the past 2 decades, illustrating improved survival and cure after CRC surgery. Moreover, median survival of the uncured population has also increased. Reasons for this could be decreased all-cause mortality, and possibly improved supportive care services (ie, palliative care).$^{24}$ In line with the ameliorated survival of patients with nonmetastasized CRC, broader performance of treatment strategies for patients with metastasized disease led to better outcomes. For instance, selected patients can be treated with chemotherapy, ablation therapy, or surgical resection of metastases. 
A
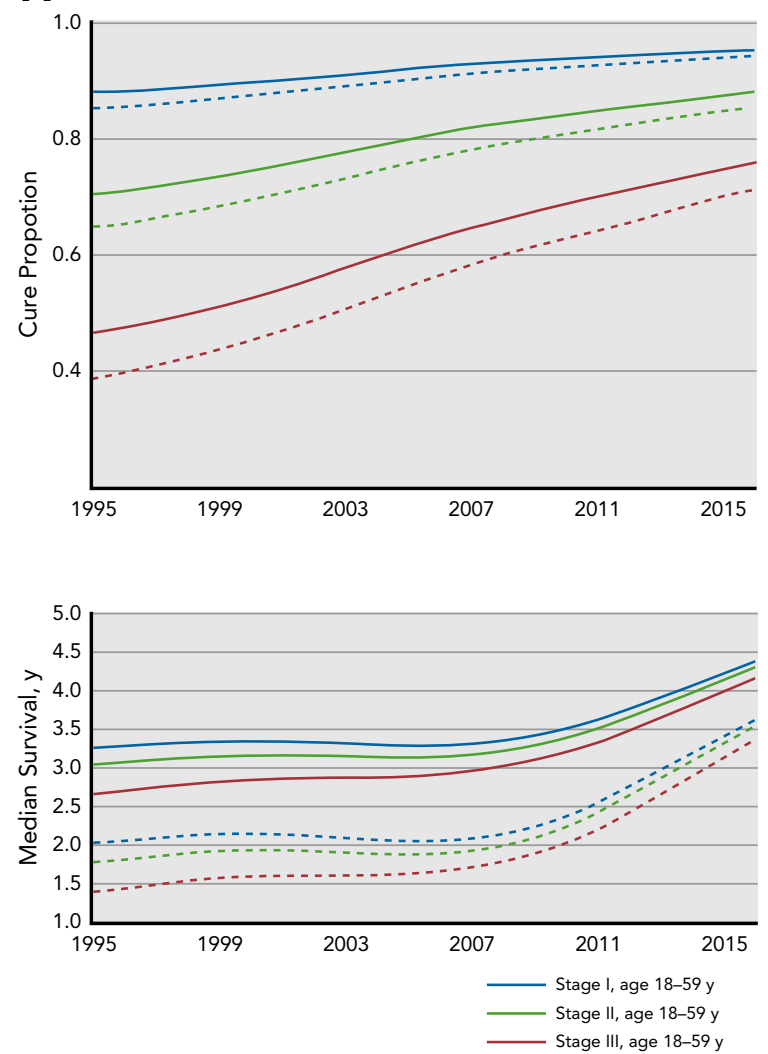

B
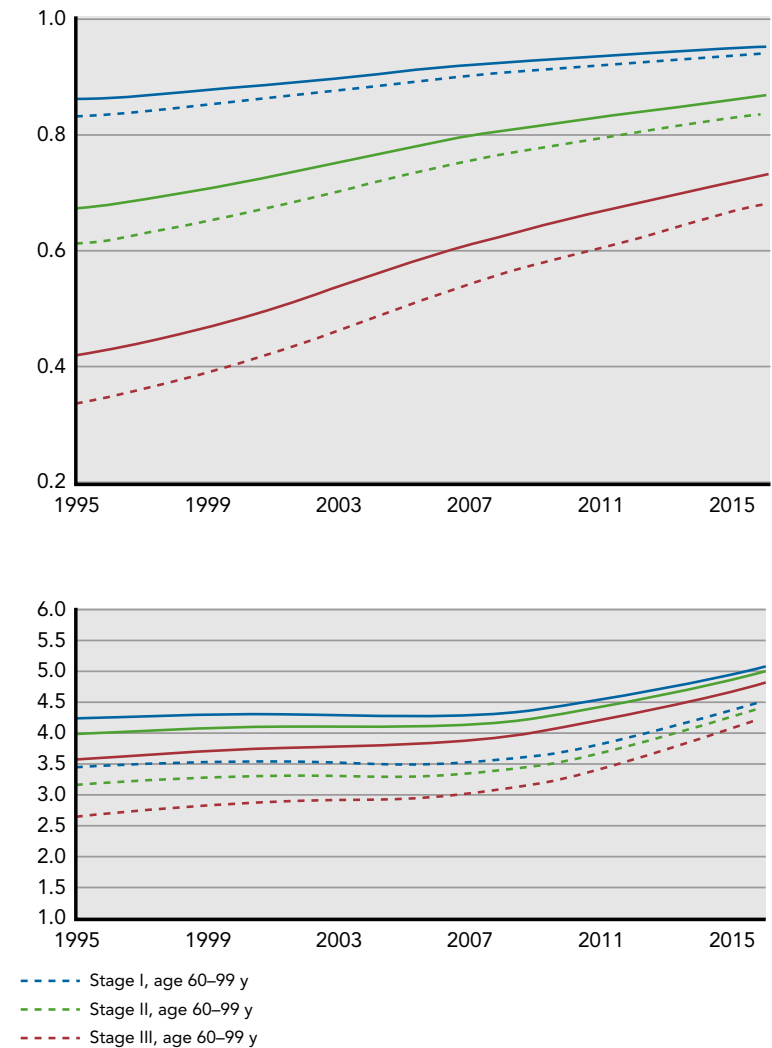

Figure 4. Cure proportions (top; ratios) and median survival (bottom; years) of the uncured among patients with stage I-III (A) colon or (B) rectal cancer diagnosed and followed between 1995 and 2016.

Relative and conditional survival outcomes presented here are slightly better than in earlier studies. ${ }^{5,11,12}$ This is possibly because of the more recent data in the present study. Only few studies have modeled cure and TTC in patients with CRC. $6,17,18$ These studies from different countries found lower cure fractions for patients with CRC ranging between 0.45 and 0.57 and longer TTC estimates between 7 and 10 years after diagnosis. The results from these studies are limited by heterogeneity. First, they often present data from $>10$ years ago. Second, results are not always stratified by disease stage, age, and sex. Third, it is common for outcomes to be presented for CRC as a group rather than for colon and rectal cancer separately. In our study, recent Dutch national data over a period of $>20$ years were used to calculate cure proportion and TTC. We strove to minimize these limitations by providing organ-, sex-, and age-specific outcomes.

Using recent data reflects the current state of survival of patients with CRC in the Netherlands. In the past decade, many advancements have been made in the diagnosis, treatment, and follow-up of patients with CRC. Broader practice of multidisciplinary treatment strategies, the change of postoperative to preoperative radiotherapy and chemotherapy, and limited measurable in this study the introduction of national prevention programs led to earlier diagnosis, disease downstaging, and less treatment-related mortality and morbidity. Including data from before this period might have biased our conditional survival outcomes. However, long-term follow-up is needed to calculate conditional survival and to determine TTC. In addition, we illustrated that the conditional survival outcome of recently diagnosed patients is better. We chose to only include endoscopically and surgically treated patients, because these remain the gold standard for curative treatment. Excluding nonresected patients (likely older, frail patients) might bias comparison with previous studies on this subject. ${ }^{5,11,12}$ However, most patients have received endoscopic or surgical treatment, and up-to-date conditional survival estimates based on patients who received similar treatment is therefore most relevant.

Another factor that might have affected our analyses is stage migration as a result of preoperative therapies. Clinically advanced disease could be downstaged in patients with good response to neoadjuvant therapy. Consequently, these patients will have lower pathologic TNM stages compared with clinical TNM stages after preoperative systemic or radiation therapy. Bosch et $\mathrm{al}^{25}$ described that survival was different between clinical and 
pathologic stage in patients with rectal cancer, and that survival outcomes are not always comparable. However, pathologic stage is a more precise measure of the extent of cancer and a better predictor for treatment response and prognosis.

Last, we presented relative survival outcomes. Relative survival is an approximation of cause-specific survival in a hypothetical world in which competing risks are assumed to be eliminated, meaning that one cannot die of causes other than the cancer of interest. Results should be communicated with caution, because daily practice could differ from the hypothetical world. However, it is useful as a measure of the survival of patients with cancer that is independent of background mortality, which makes comparisons across time and different age groups possible. The assumptions made in the relative survival framework are strong, and relative survival provides accurate estimates of cancer-specific survival. ${ }^{26}$

\section{Conclusions}

This study presented relative survival, conditional survival, cure proportions, and TTC of patients with stage I-III colon or rectal cancer. Our results can be generalized to other Western countries with comparable healthcare systems. In an era of minimally invasive and multidisciplinary treatment strategies, the results of this comprehensive study provide clinically highly relevant, up-to-date survival outcome information for clinicians, patients, and health policymakers.

\section{Acknowledgments}

We thank Marissa van Maaren (Netherlands Comprehensive Cancer Organization) and Sarwar Islam Mozumder (University of Leicester) for their valuable comments regarding the statistical commands used in this study.

Submitted January 7, 2020; accepted for publication March 27, 2020.

Author contributions: Study concept: Qaderi, de Wilt, Verhoeven. Study design: All authors. Data acquisition: Qaderi, Verhoeven. Quality control of data and algorithms: Qaderi, Verhoeven. Data analysis and interpretation: Qaderi, Verhoeven, Dickman. Statistical analysis: All authors. Manuscript preparation, editing, and review: All authors.

Disclosures: Dr. Verhoeven has disclosed that he has received grant/ research support from Roche and Bristol-Myers Squibb. The remaining authors have disclosed that they have not received any financial consideration from any person or organization to support the preparation, analysis, results, or discussion of this article.

Correspondence: Seyed M. Qaderi, MD, PhD, Department of Surgical Oncology, Radboud University Medical Center, Geert Grooteplein Zuid 10, 6525 GA Nijmegen, the Netherlands.

Email: seyed.qaderi@radboudumc.nl

\section{References}

1. Kuipers EJ, Grady WM, Lieberman D, et al. Colorectal cancer. Nat Rev Dis Primers 2015;1:15065.

2. Dutch Comprehensive Cancer Network. Cancer Statistics Netherlands. Accessed November 4, 2019. Available at: http://www.cijfersoverkanker.nl/

3. van der Stok EP, Spaander MCW, Grünhagen DJ, et al. Surveillance after curative treatment for colorectal cancer. Nat Rev Clin Oncol 2017;14: 297-315.

4. Brouwer NPM, Heil TC, Olde Rikkert MGM, et al. The gap in postoperative outcome between older and younger patients with stage I-III colorectal cancer has been bridged: results from the Netherlands cancer registry. Eur J Cancer 2019;116:1-9.

5. van Erning FN, van Steenbergen LN, Lemmens VEPP, et al. Conditional survival for long-term colorectal cancer survivors in the Netherlands: who do best? Eur J Cancer 2014;50:1731-1739.

6. Andersson TM, Dickman PW, Eloranta S, et al. Estimating and modelling cure in population-based cancer studies within the framework of flexible parametric survival models. BMC Med Res Methodol 2011;11:96.

7. Belot A, Ndiaye A, Luque-Fernandez MA, et al. Summarizing and communicating on survival data according to the audience: a tutorial on different measures illustrated with population-based cancer registry data. Clin Epidemiol 2019;11:53-65.

8. van Maaren MC, Strobbe LJA, Smidt ML, et al. Ten-year conditional recurrence risks and overall and relative survival for breast cancer patients in the Netherlands: taking account of event-free years. Eur J Cancer 2018; 102:82-94.

9. Othus $\mathrm{M}$, Barlogie B, Leblanc ML, et al. Cure models as a useful statistical tool for analyzing survival. Clin Cancer Res 2012;18:3731-3736.

10. Cancer in Norway: cancer incidence, mortality, survival and prevalence in Norway. Accessed June 22, 2020. Available at: https://www.kreftregisteret.no/globalassets/cancer-in-norway/2018/cin2018.pdf

11. van Steenbergen LN, Steur M, Lemmens VE, et al. Minimal excess mortality for long-term colon cancer survivors in the Netherlands 1989-2008. Eur J Cancer 2013;49:585-592.

12. Drouillard $A$, Bouvier $A M$, Rollot $F$, et al. Conditional net survival: relevant prognostic information for colorectal cancer survivors. A French population-based study. Dig Liver Dis 2015;47:597-601.
13. Schouten LJ, Jager JJ, van den Brandt PA. Quality of cancer registry data: a comparison of data provided by clinicians with those of registration personnel. Br J Cancer 1993;68:974-977.

14. Ederer $\mathrm{F}$, Heise $\mathrm{H}$. Instructions to IBM 650 Programmers in Processing Survival Computations. Methodological Note No. 10, End Results Evaluation Section. Bethesda, MD: National Cancer Institute; 1959.

15. Seppä K, Hakulinen T, Läärä $E$, et al. Comparing net survival estimators of cancer patients. Stat Med 2016;35:1866-1879.

16. Dickman PW. Conditional survival. Accessed March 16, 2020. Available at: http://pauldickman.com/software/stata/conditional-survival/

17. Dal Maso L, Guzzinati S, Buzzoni C, et al. Long-term survival, prevalence, and cure of cancer: a population-based estimation for 818902 Italian patients and 26 cancer types. Ann Oncol 2014;25:2251-2260.

18. Boussari $O$, Romain $G$, Remontet $L$, et al. A new approach to estimate timeto-cure from cancer registries data. Cancer Epidemiol 2018;53:72-80.

19. Andersson TML, Lambert PC. Fitting and modeling cure in populationbased cancer studies within the framework of flexible parametric survival models. Stata J 2012;12:623-638

20. Dickman PW, Coviello E. Estimating and modeling relative survival. Stata J 2015;15:186-215.

21. Lambert PC. Modeling of the cure fraction in survival studies. Stata J 2007; 7:351-375.

22. von Elm E, Altman DG, Egger M, et al. The Strengthening the Reporting of Observational Studies in Epidemiology (STROBE) statement: guidelines for reporting observational studies. Lancet 2007;370:1453-1457.

23. Brouwer NPM, Bos ACRK, Lemmens VEPP, et al. An overview of 25 years of incidence, treatment and outcome of colorectal cancer patients. Int $J$ Cancer 2018;143:2758-2766.

24. Verdecchia A, De Angelis $R$, Capocaccia $R$, et al. The cure for colon cancer results from the EUROCARE study. Int J Cancer 1998;77:322-329.

25. Bosch SL, Verhoeven RHA, Lemmens VEPP, et al. Type of preoperative therapy and stage-specific survival after surgery for rectal cancer: a nationwide population-based cohort study. Virchows Arch 2019;475:745-755

26. Lambert PC, Dickman PW, Nelson CP, et al. Estimating the crude probability of death due to cancer and other causes using relative survival models. Stat Med 2010;29:885-895. 